\title{
Rheodynamic Lubrication of an Externally Pressured Thrust Bearing Using Herschel-Bulkley Fluid with Sinusoidal Injection
}

\author{
I. J. Amalraj ${ }^{1}$, S.Narasimman ${ }^{1}$ and A. Kandasamy ${ }^{2 \dagger}$. \\ ${ }^{1}$ Department of Mathematics, SSN College of Engineering, Kalavakkam-603110 Chennai, India \\ ${ }^{2}$ Professor, Department of Mathematical and Computational Sciences, National Institute of Technology Karnataka, \\ Surathkal, Mangalore-575025, India. \\ †Corresponding Author Email: kandy@nitk.ac.in
}

(Received December 1, 2010; accepted July 10, 2011)

\begin{abstract}
Lubricants with variable viscosity are assuming greater importance for its application in polymer industry, thermal reactors and in biomechanics. With the bearing operations in machines being subject to high speeds, loads, increasing mechanical shearing forces and continually increasing pressure, there has been an increasing interest to use nonNewtonian fluids characterized by a yield value. Some of them, which fit into this class, are Bingham, Casson and Herchel-Bulkley models. In the present work, the problem of an externally pressurized thrust bearing lubricated with Herschel-Bulkley fluid under the sinusoidal flow rate has been investigated. Herschel-Bulkley fluids are characterized by a yield value, which leads to the formation of rigid core in the flow region. The shape and extent of the core has been determined numerically for various values of the Herschel-Bulkley number, power-law index, amplitude of sinusoidal fluid film and time. Numerical solutions have been obtained for the bearing performances such as pressure distribution and load capacity for different values of the Herschel-Bulkley number, power-law index, amplitude of sinusoidal fluid film and time. The effects of sinusoidal injection of the lubricant and the non-Newtonian characteristics on the bearing performances have been discussed.
\end{abstract}

Keywords: Non-Newtonian lubricant, Herschel-Bulkley fluids, Yield stress, Externally pressurized thrust bearing, Sinusoidal injection.

\section{INTRODUCTION}

As the bearing operations in machines are subjected to high speed, load, increasing mechanical shearing forces and continually increasing pressures, there has been an increasing interest in the usage of non-Newtonian fluids with yield stress as lubricants, like, Bingham plastic, Casson and Herschel-Bulkley fluids. The HerschelBulkley fluids include both shear thinning and shear thickening materials. They represent the combination of Bingham plastic and power-law fluids and are also known as yield-power law fluids. Examples of such materials are greases, colloidal suspensions, starch pastes and blood flow through narrow tubes.

Many researchers have analyzed the rheodynamic lubrication in the externally pressurized thrust bearing by considering both Newtonian and non-Newtonian fluids. Roy et al. (1993) have discussed the effect of inertial forces in the externally pressurized bearing by considering the visco-elastic fluid as the lubricant. Elsharkawy et al. (1996) have studied the effects of a porous layer on the hydrodynamic lubrication of an externally pressurized thrust bearing and obtained the numerical solutions of pressure distribution and load capacity. Jaw-Ren Lin (1999) has investigated the combined effects of couple stress, fluid inertia and recess volume fluid compressibility on the steady and dynamic state characteristics of hydrostatic circular step thrust bearings. The problem of an externally pressurized thrust bearing lubricated with Herschel Bulkley fluids has been considered by Kandasamy et al. (2006) and numerical solutions has been obtained for the pressure distribution and the load capacity.

Using the Herschel-Bulkley fluids as lubricants, the problems of different types of bearings have been investigated by many researchers. Squeeze flow experiments have been carried out by Sherwood et al. (1998) for a Herschel Bulkley fluid using lubricated wall boundary conditions. Chan et al. (2002) have analyzed the squeezing flow of the Herschel-Bulkley fluid and obtained the deviations of the simulated finite element results, by using the lubrication approximation, with that of experimental results. Both theoretical and experimental investigation of the force required to squeeze the Herschel-Bulkley material without slip for different surface patterns has been carried out by Meeten (2005). The behavior of the Herschel-Bulkley fluids between two ellipsoid rollers has been analyzed by Zhang et al. (2005). The rheodynamic lubrication of 
the squeeze film bearing using Herschel -Bulkley fluids has been analyzed by Kandasamy et al. (2006).

Hashimoto et al. (1986) have examined the bearing performances in a squeeze film bearing with sinusoidal motion lubricated using a power law fluids. With sinusoidal squeeze motion, Usha et al. (2002) have applied the energy integral approach to find the behaviour of curved squeeze film bearing using Newtonian lubricant. Kandasamy et al. (2007) have discussed the effects of fluid inertia, non-Newtonian characteristics and the amplitudes of sinusoidal squeeze motion on the bearing performances of a squeeze film bearing using Bingham fluid. Recently, Amalraj et al. (2010) have analyzed the bearing performances in an externally pressurized thrust bearing using Bingham fluids with sinusoidal injection of the lubricant.

In the present work, the problem of an externally pressurized thrust bearing lubricated with HerschelBulkley fluid under the sinusoidal flow rate has been analyzed. During the operations of the bearings the maximum viscous shearing stresses arise in the region between the plates. Therefore, there may be a region in the film where the shearing stresses do not exceed the yield value of the lubricant and thereby a core with zero velocity gradients is formed. The flow occurs only in the region where the shear stress exceeds the yield value. The shape and extent of the core has been determined numerically for various values of the Herschel-Bulkley number, power-law index, amplitude and time for the case of sinusoidal flow rate. The flow is confined to the region between the core and the circular plates of the bearing. Numerical solutions have been obtained for the bearing performances such as pressure distribution and load capacity for different values of the Herschel-Bulkley number and power-law index and for various amplitudes of the sinusoidal feeding. The characteristic of an externally pressurized thrust bearing has been investigated through NonNewtonian effects and the sinusoidal conditions.

\section{MAThematical Formulation OF THE PROBLEM}

The geometry of the problem is shown in Fig.1

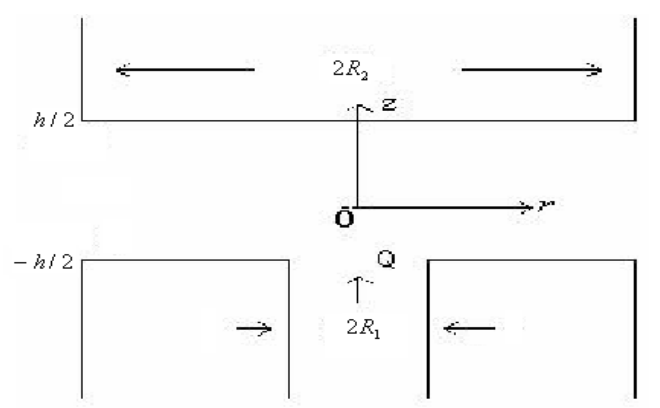

Fig. 1. Geometry of an externally pressurized thrust bearing

We consider an isothermal incompressible steady flow of the time-independent Herschel-Bulkley fluid injected between two circular plates, separated by a distance ' $h$ '. Let $R_{1}$ be the radius of the film inlet, $R_{2}$ be the radius of the film outlet. Let $\mathrm{p}$ denote the pressure of the film, $p_{a}$ the atmospheric pressure and $\rho$ the density of the fluid. The cylindrical polar co-ordinates $(r, \theta, z)$ with axial symmetry have been considered. The origin is fixed at the centre of the plate, $r$ measuring the distance along the radial direction and $z$ along the axis normal to the bearing. Let $v_{r}$ and $v_{z}$ be velocity components along $r$ and $z$ directions respectively.

The constitutive equation for these fluids is given by Whorlow (1980)

$\tau=\eta_{2}+\eta_{1}|\dot{\gamma}|^{n}$

where $\tau$ is the shear stress $\eta_{2}$ is the yield stress, $\eta_{1}$ is called the coefficient of fluidity, $|\dot{\gamma}|$ is the rate of strain and $\mathrm{n}$ is the power-law index. In those regions of the film, where the shear stress is less than the yield value, there will be a core formation which will move with constant velocity, $v_{c}$. Let the boundaries of the core be given by $z=-\delta(r) \frac{h}{2}$ and $z=+\delta(r) \frac{h}{2}$ as shown in

Fig. 2.

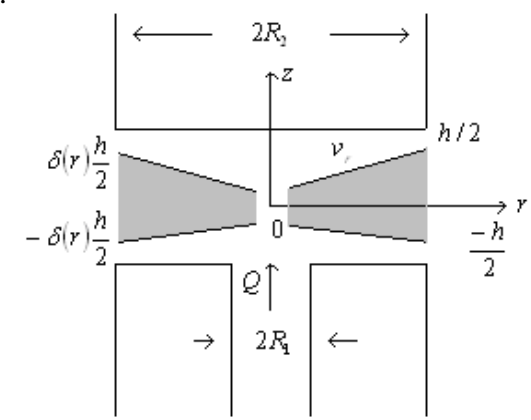

Fig. 2. Shape of the core in an externally pressurized thrust bearing

Applying the basic assumptions of lubrication theory for thin films, the governing equations for the above system is given by:

$-\frac{\partial p}{\partial r}=\frac{\partial \tau_{r z}}{\partial z}$

$\frac{\partial p}{\partial z}=0$

$\frac{1}{r} \frac{\partial}{\partial r}\left(r v_{r}\right)+\frac{\partial v_{z}}{\partial z}=0$

$\tau_{r z}=\eta_{2} \pm \eta_{1}\left|\frac{\partial v_{r}}{\partial v_{z}}\right|^{n}$

The Eqs. (2), (3) and (5) together with continuity, Eq. (4), are to be solved under the following boundary conditions:

$v_{r}=0$ at $z= \pm \frac{h}{2}$ 
$v_{r}=v_{c}$ at $z= \pm \delta(r) \frac{h}{2}$

$\frac{\partial v_{r}}{\partial z}$ is continuous, at $\tau=\eta_{2}$

$p=p_{a}$ at $r=R_{2}$

where $v_{c}$ is the core velocity and $p_{a}$ is atmospheric pressure.

\section{Solution of the Problem}

Integrating Eq. (2) and using Eq. (5) and the boundary conditions (6) and (7), the velocity distribution in the flow region is obtained as

$v_{r}=\left(\frac{n}{n+1}\right)\left(\frac{1}{\eta_{1}}\right)^{\frac{1}{n}}\left(\frac{-d p}{d r}\right)^{\frac{1}{n}}\left[\left(z-\frac{\delta h}{2}\right)^{\frac{1}{n}+1}-\left(\frac{h}{2}-\frac{\delta h}{2}\right)^{\frac{1}{n}+1}\right]$

The velocity of the plug core is given by

$v_{c}=-\left(\frac{n}{n+1}\right)\left(\frac{1}{\eta_{1}}\right)^{\frac{1}{n}}\left(\frac{-d p}{d r}\right)^{\frac{1}{n}}\left(\frac{h}{2}-\frac{\delta h}{2}\right)^{\frac{1}{n}+1}$

$0 \leq z \leq \frac{\delta h}{2}$

The equation of conservation of mass which depends on the bearing configuration in this case is

$$
Q=4 \pi r \int_{0}^{h / 2} v_{r} d z
$$

where $Q$ is the flow rate per unit width.

Using (10) and (11) in Eq. (12) we get

$$
\begin{array}{r}
Q=-\left[\frac{n}{(n+1)(2 n+1)}\right]\left(\frac{1}{\eta_{1}}\right)^{\frac{1}{n}} \frac{\pi r h^{\frac{1}{n}+2}\left(\frac{-d p}{d r}\right)^{\frac{1}{n}}}{2^{\frac{1}{n}}} \times \\
(1-\delta)^{\frac{1}{n}+1}(n \delta+n+1)
\end{array}
$$

Now considering the equilibrium of an element in the yield surface, $-\frac{\delta h}{2} \leq z \leq+\frac{\delta h}{2}$, it is found that

$$
\frac{d p}{d r}=\frac{2 \eta_{2}}{\delta(r) h}
$$

Eliminating the pressure gradient from Eqs. (13) and (14), we get an algebraic equation for determining the thickness of the core $\delta(r)$ as

$$
\frac{\left[\frac{(n+1)(2 n+1)}{n}\right] Q \eta_{1}^{\frac{1}{n}}}{\pi r h^{2} \eta_{2}^{\frac{1}{n}}}=\frac{(1-\delta)^{\frac{1}{n}}(n \delta+n+1)}{\delta^{\frac{1}{n}}}
$$

Again from Eq. (13), $\frac{d p}{d r}=\frac{2\left[\frac{(n+1)(2 n+1)}{n}\right]^{n} Q^{n} \eta_{1}}{\pi^{n} r^{n} h^{2 n+1}(1-\delta)^{n+1}(n \delta+n+1)^{n}}$

Let us introduce the sinusoidal (flow rate) feeding of the lubricant as

$Q=Q_{0}+a \sin \left(\omega_{f} t\right)$

which influences the sinusoidal fluid film between the plates. Here, $Q_{0}$ is the mean flow rate, $a$ is the amplitude of the flow rate variation, $\omega_{f}$ is the frequency of oscillation and $t$ is the time of oscillation.

The following non-dimensional parameters are introduced:

$$
r^{*}=\frac{r}{R_{2}} ; \quad \delta^{*}=\delta\left(r^{*}\right) ; \quad p^{*}=\frac{p}{\left(\frac{Q_{0}{ }^{n} \eta_{1}}{\pi h^{3 n}}\right)} ; Q^{*}=\frac{Q}{Q_{0}}
$$

$A=\frac{a}{Q_{0}} ; T=\omega_{f} t \quad N=\frac{\pi R_{2} h^{2}}{Q_{0}}\left(\frac{\eta_{2}}{\eta_{1}}\right)^{\frac{1}{n}}$

Using the above non-dimensional quantities, Eqs. (15) and (16) take the form

$\frac{\left[\frac{(n+1)(2 n+1)}{n}\right][1+A \sin (T)]}{N r^{*}}=\frac{\left(1-\delta^{*}\right)^{\frac{1}{n}+1}\left(n \delta^{*}+\delta^{*}+1\right)}{\left(\delta^{*}\right)^{\frac{1}{n}}}$

in which $\mathrm{N}$ is the Herschel-Bulkley number.

The core thickness can be determined from the algebraic Eq. (19). The root $\delta *(N, n, A, T, r *)$ of the Eq. (19), which is positive and less than unity, determines the shape of the yield surface. The value of the root, for a particular material (i.e) for a given Herschel-Bulkley number and the power-law index, is obtained for various values of $r^{*}, A$ and $T$ using any numerical iterative technique. Knowing $\delta\left(r^{*}\right)$, the pressure distribution can be obtained from (19) by integrating it numerically using the boundary condition (9) and is given by

$p^{*}-p_{a}^{*}=\int_{r *}^{1}\left(\frac{d p^{*}}{d r^{*}}\right) d r^{*}$

Then, the load carrying capacity of the externally pressurized thrust bearing can be obtained by integrating the pressure along the radius of the bearing and is given by

$W=\int_{R *}^{1}\left(p^{*}-p_{a}^{*}\right) r * d r *$

where $R^{*}=\frac{R_{1}}{R_{2}}$ is the ratio of inside to outside radius of the bearing. This integration is performed numerically for various values of Herschel-Bulkley number, power-law index, amplitude and time. 
I.Jayakaran Amalraj et al. / JAFM, Vol. 5, No. 4, pp. 71-79, 2012.

\section{RESUlTS AND DiscUSSION}

The behaviour of core thickness $\delta *$ for various values of amplitude $(A)$, time $(T)$ and non-Newtonian characteristics $(N, n)$ at every point of radius $\left(r^{*}\right)$ is computed and the results are given in Figs. 3-8.

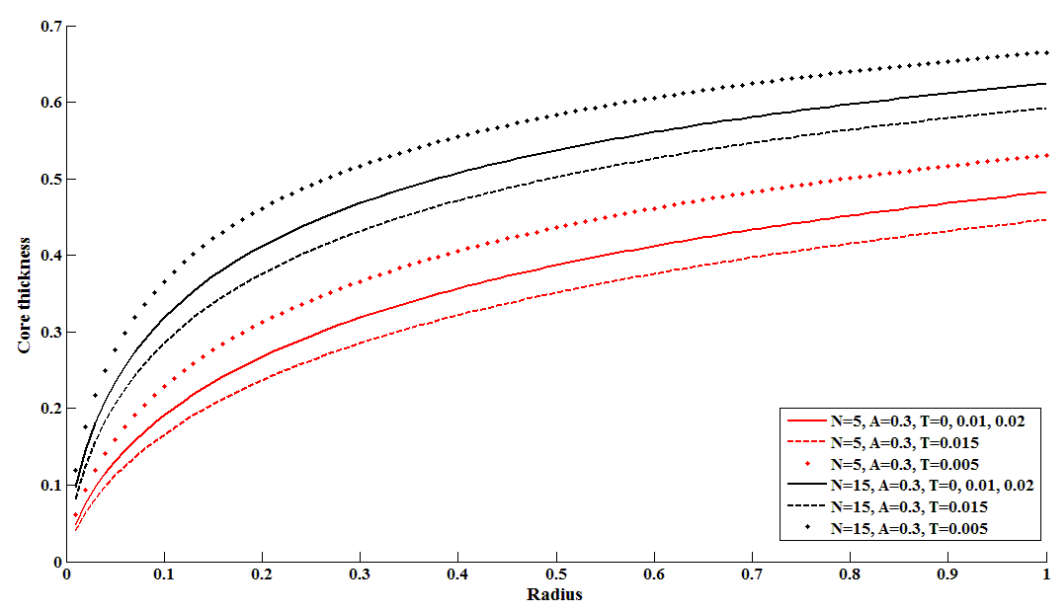

Fig. 3. Core thickness variation along the radius for $A=0.3: n=0.7$

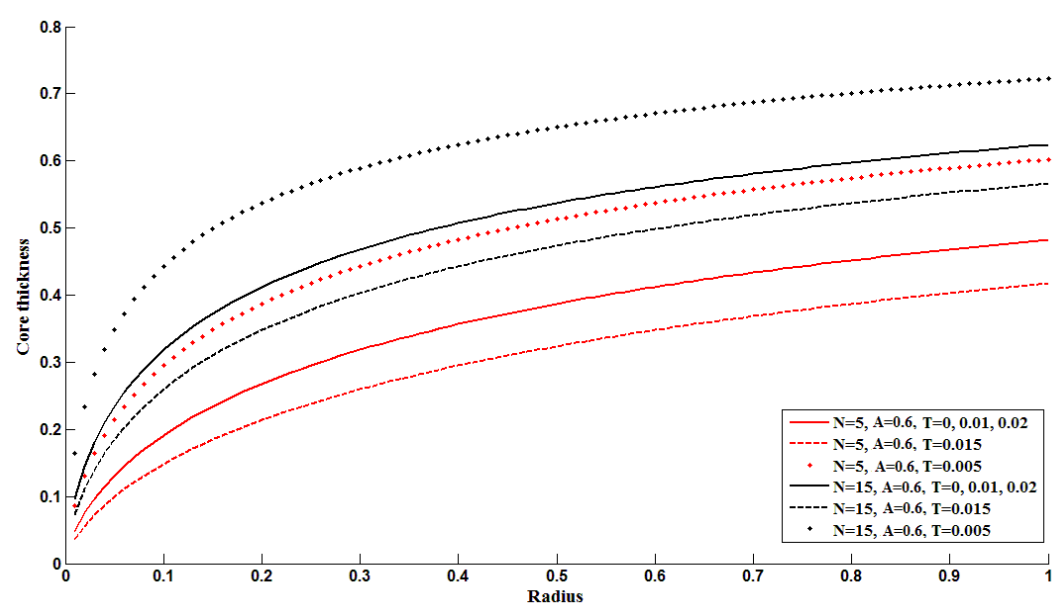

Fig. 4. Core thickness variation along the radius for $A=0.6: n=0.7$

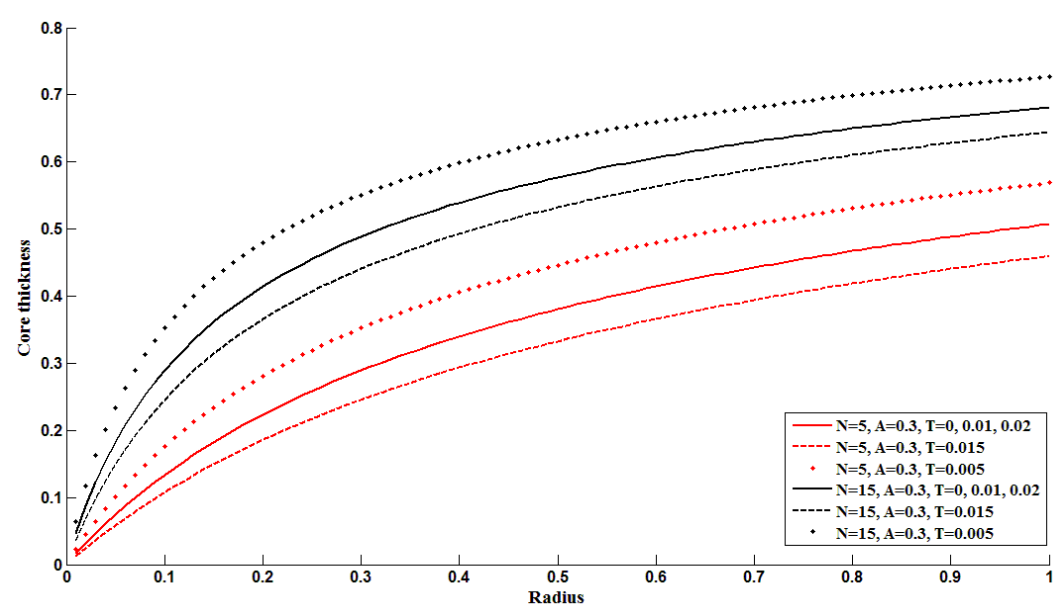

Fig. 5. Core thickness variation along the radius for $A=0.3: n=1.0$ 


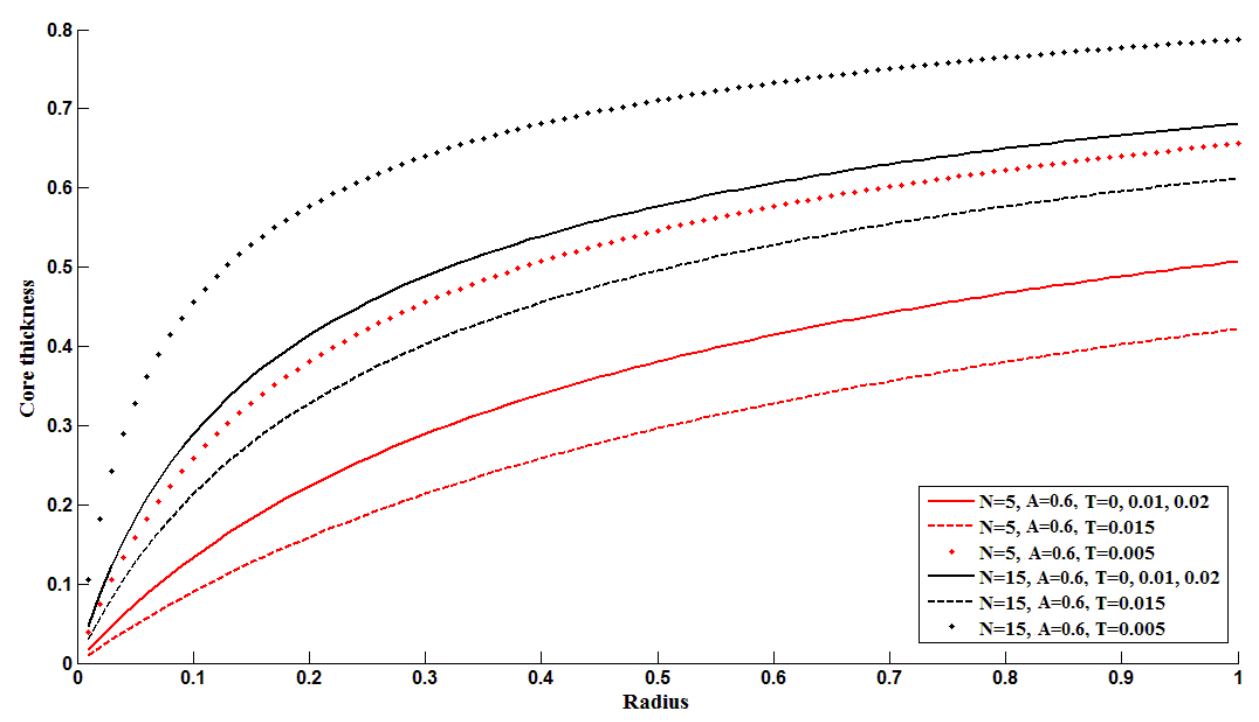

Fig. 6. Core thickness variation along the radius for $A=0.6: n=1$

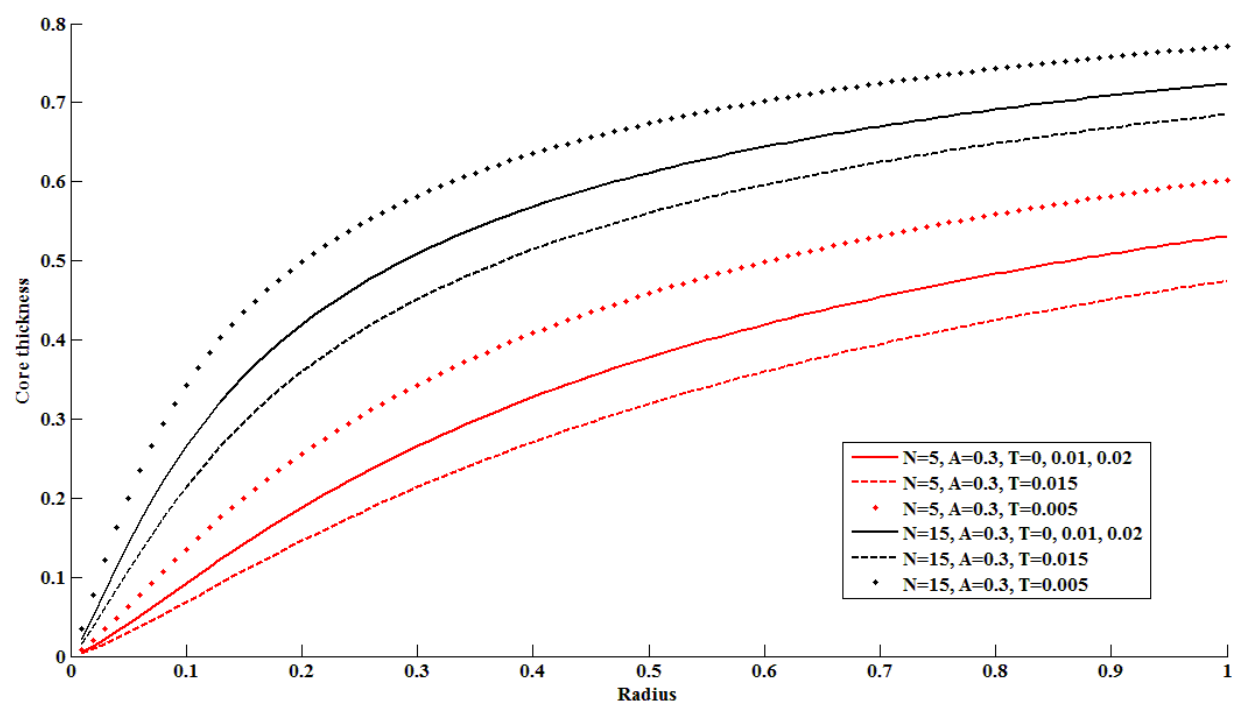

Fig. 7. Core thickness variation along the radius for $A=0.3: n=1.3$

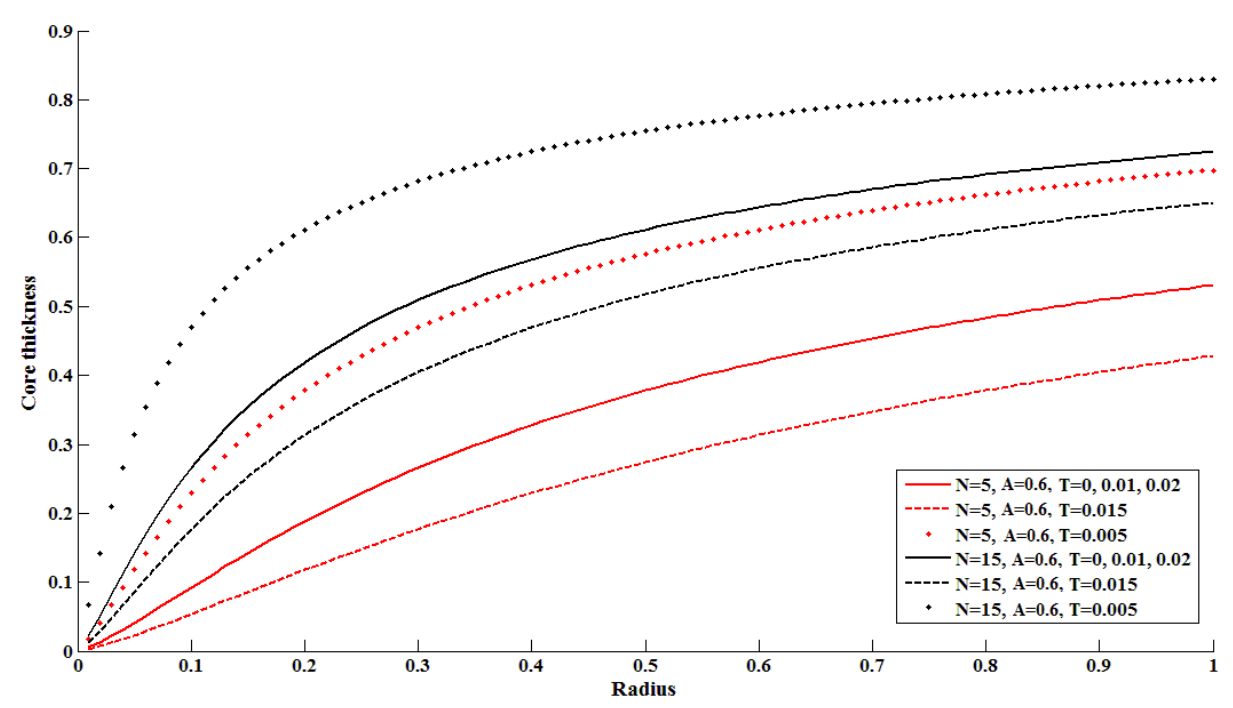

Fig. 8. Core thickness variation along the radius for $A=0.6: n=1.3$ 
The core thickness is found to be minimal at the center of the plates and increases towards the periphery. It has been observed that the thickness of the core increases when the Herschel-Bulkley number increases for a constant power-law index, amplitude and time. Similarly, the thickness of the core increases with the increase in power-law index, for a constant HerschelBulkley number, amplitude and time. However, with respect to time, the formation of plug core decreases only up to an optimum time, after which it increases gradually before changing its course for a fixed amplitude, power-law index and Herschel-Bulkley number. The impact of increase in amplitude on core thickness is also found to increase marginally for any particular Herschel-Bulkley number and power-law index.

The radial distribution of film pressure along the radius of the thrust bearing for different values amplitude $(A)$, time $(T)$ and Herschel-Bulkley numbers $(N)$ and powerlaw index $(n)$ are computed and shown in Figs. 9-13.

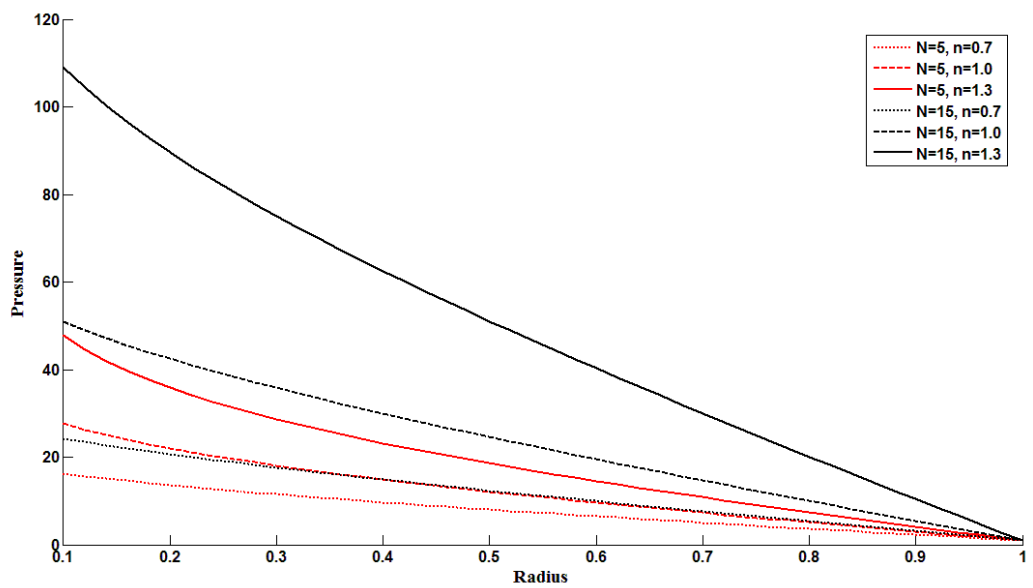

Fig. 9. Pressure distribution along radius when $T=0 \& A=0.3$

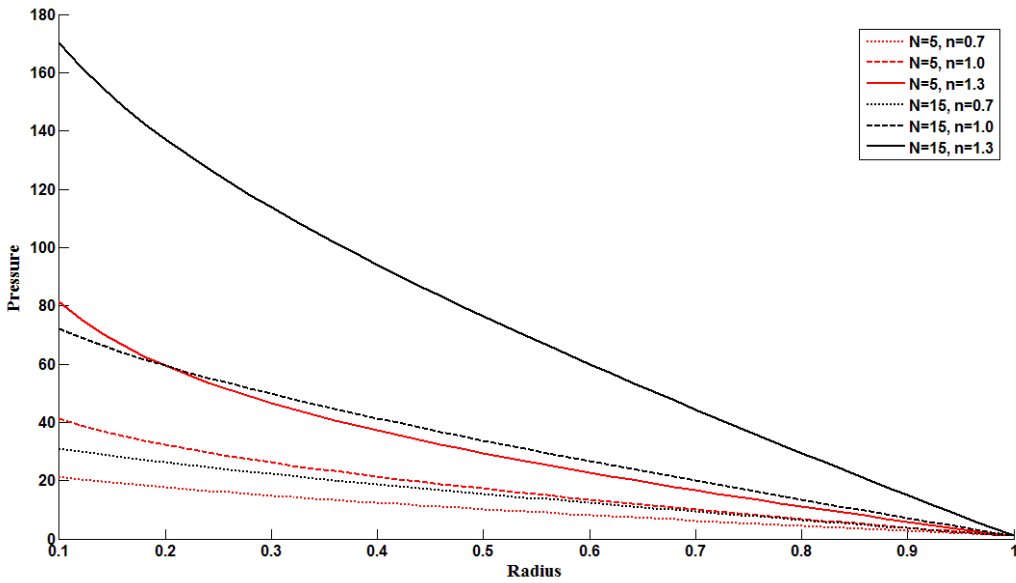

Fig. 10. Pressure distribution along radius when $T=0.005 \& A=0.3$

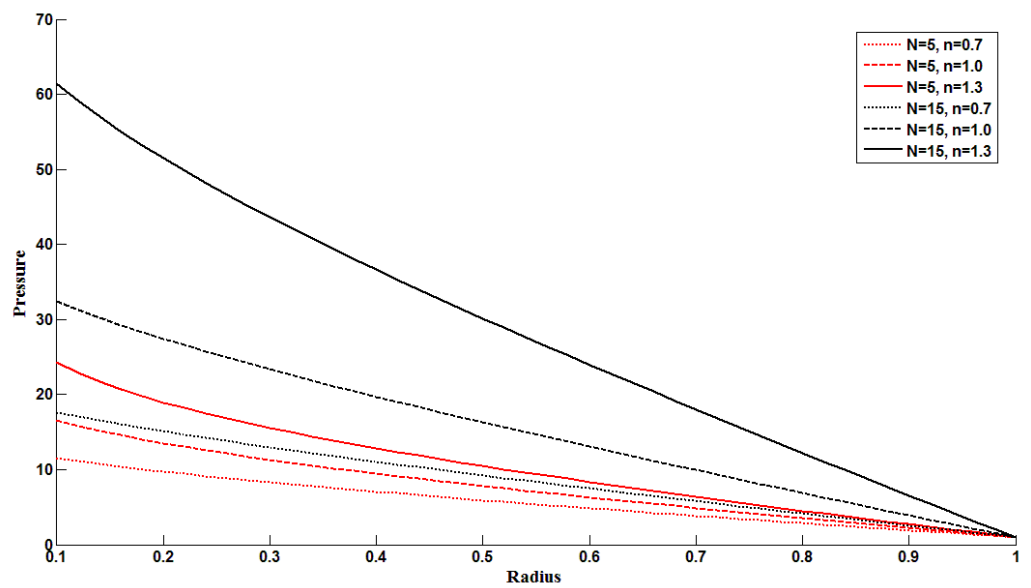

Fig. 11. Pressure distribution along radius when $T=0.015 \& A=0.3$ 


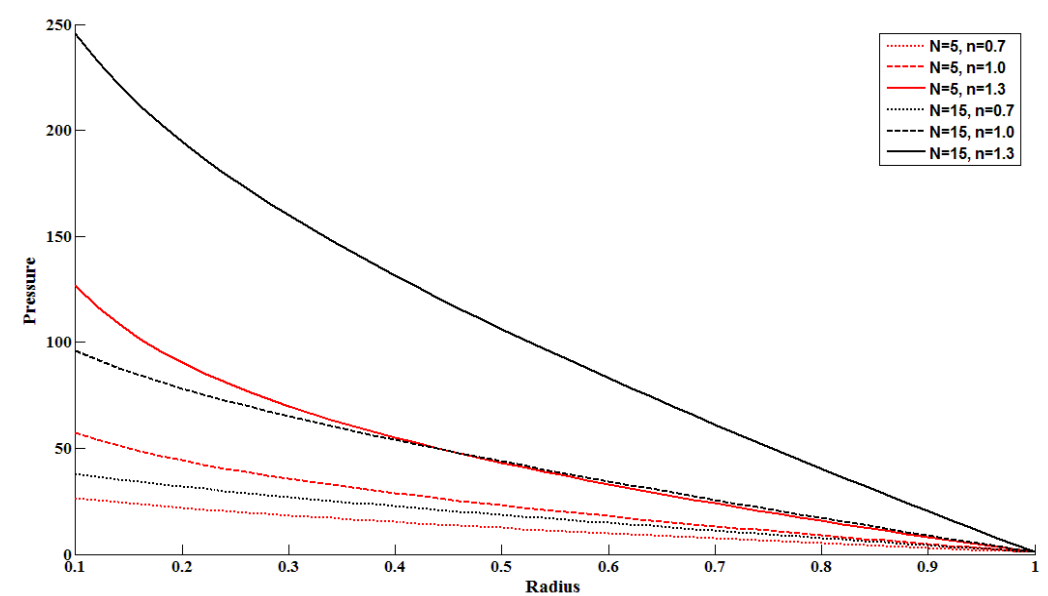

Fig. 12. Pressure distribution along radius when $T=0.005 \& A=0.6$

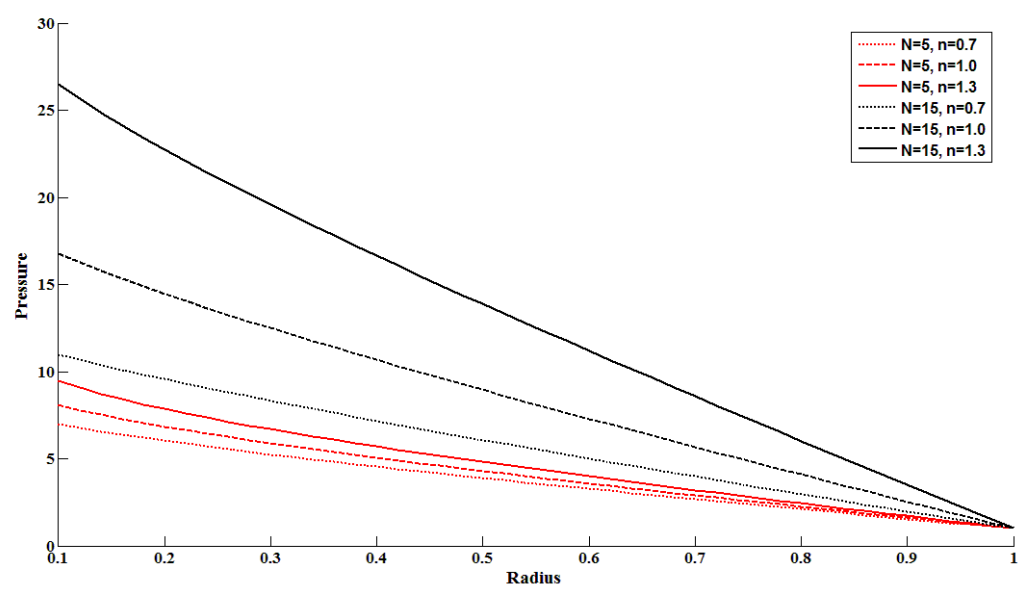

Fig. 13. Pressure distribution along radius when $T=0.015 \& A=0.6$

The pressure is found to decrease from centre to periphery of the bearing. It is observed that the pressure of the fluid film increases with the increase in HerschelBulkley number $(N)$ for a constant power-law index $(n)$, amplitude $(A)$ and time $(T)$. Also, there is a significant increase in the pressure as the power-law index increases at a given Herschel-Bulkley number, amplitude and time. For a particular Herschel-Bulkley number, power-law index and time, when the amplitude of the sinusoidal fluid film increases, the pressure is found to increase only up to an optimum time. After that the pressure is found to decrease with the increase in amplitude, before it changes its course with respect to time.

The velocity profile along the axial direction $(z *)$ for various values of the radius $\left(r^{*}\right)$ and Herschel-Bulkley numbers $(N)$, at a constant amplitude $(A)$, time $(T)$ and power-law index $(n)$ are depicted in the Fig. 14. The shape of the core, as observed earlier, is reflected in this profile. In the limiting case as $N$ tends to zero, the velocity profile is that of a parabola, which represents a Newtonian fluid.

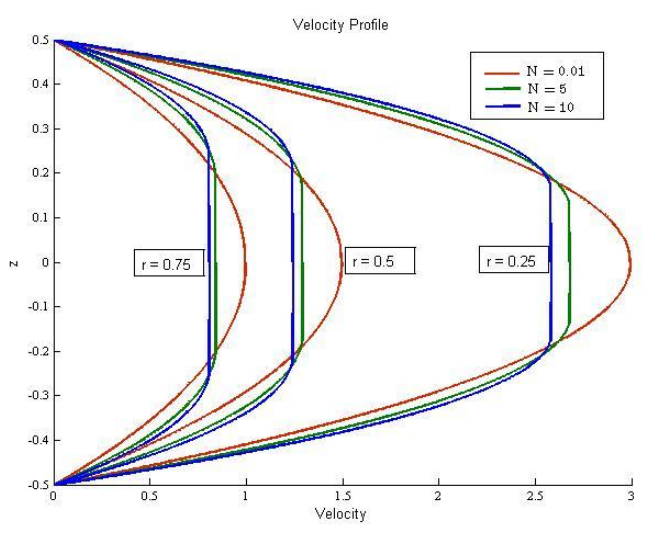

Fig. 14. Velocity profile 
I.Jayakaran Amalraj et al. / JAFM, Vol. 5, No. 4, pp. 71-79, 2012.

The results of load capacity calculated for various values of Herschel-Bulkley numbers $(N)$, power-law

index $(n)$, amplitudes $(A)$ and time $(T)$ are graphically shown in Figs. 15-17.

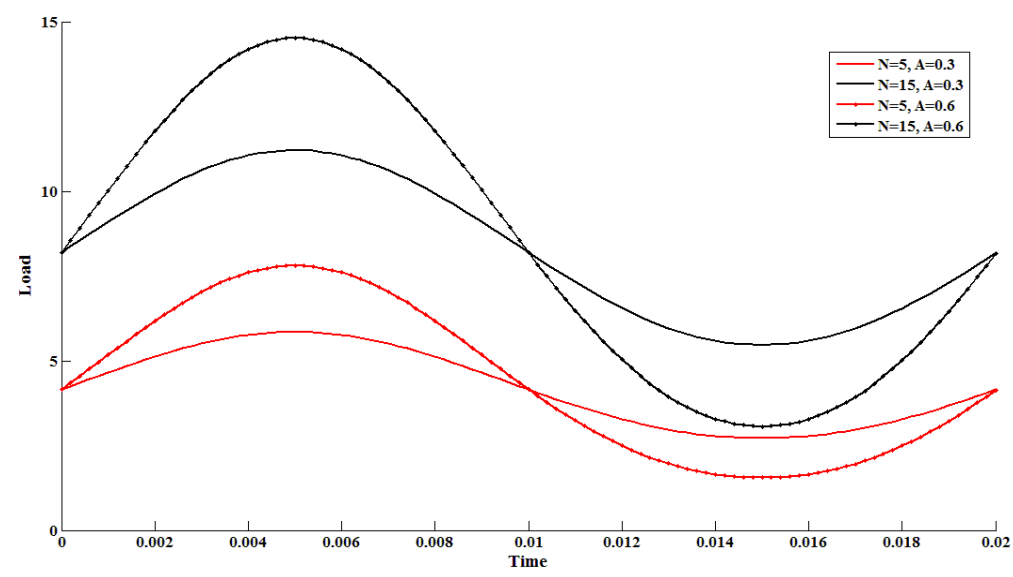

Fig. 15. Load capacity varying with time for $n=0.7$

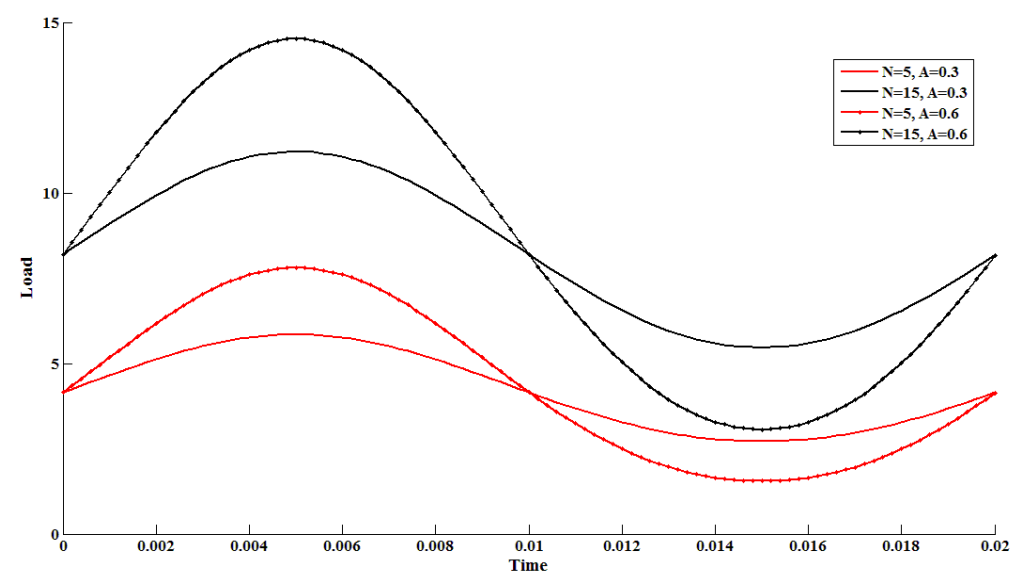

Fig. 16. Load capacity varying with time for $n=1.0$

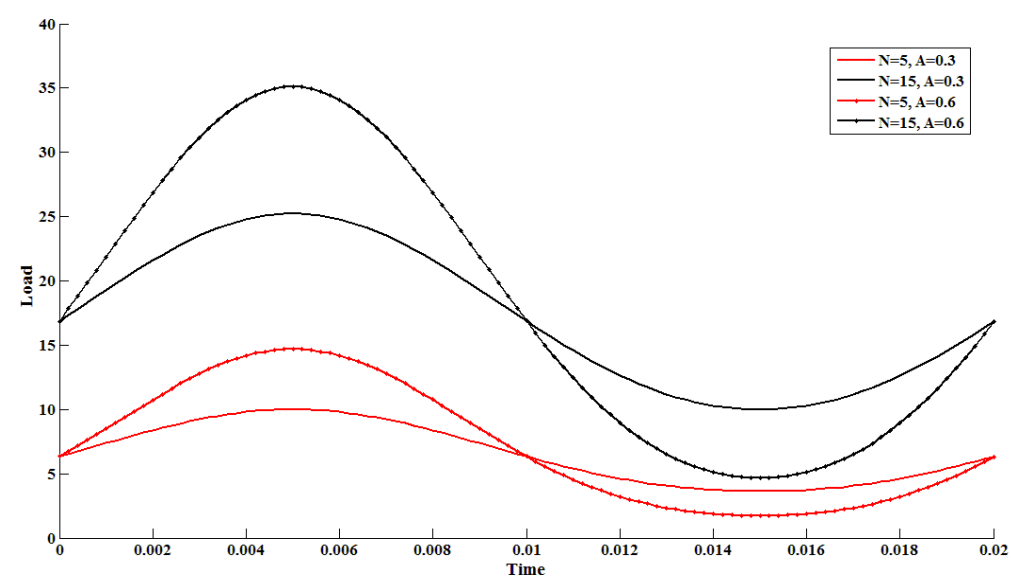

Fig. 17. Load capacity varying with time for $n=1.3$

We find that the load capacity of the bearing increases significantly with the increase in Herschel-Bulkley number for a constant power-law index and amplitude. Further, the rate of increase of the load capacity due to the increase in power-law index is found to be significant at high amplitudes. Moreover, for a constant amplitude and Herschel-Bulkley number, the load capacity of the bearing increases with the increase of time up to an optimum point beyond which it starts decreasing gradually before it changes its nature. The effects of non-Newtonian characteristics on the load capacity are more significant as the amplitude of the sinusoidal motion increases. Since, there are no results available in the literature for the lubrication of an 
externally pressurized thrust bearing with HerschelBulkley fluids under sinusoidal conditions, the present results are compared with the particular cases available in literature. For the constant flow rate, our results are in agreement with the results obtained earlier by Kandasamy et al. (2006). Further, the results corresponding to the power-law index $n=1$, in our analysis, are compared with the case of Bingham lubrication under sinusoidal condition and are found to be in agreement (Amalraj et al. 2010).

\section{CONCLUSION}

The problem of an externally pressurized thrust bearing lubricated with Herschel-Bulkley fluid under the sinusoidal flow rate has been investigated here. From the analysis, the following conclusions are drawn:

1. The core thickness of the lubricant is high for fluids with high $\mathrm{H}-\mathrm{B}$ number as well as for fluids with high power-law index.

2. The impact of 'increase in amplitude' on core thickness is found to increase marginally for a lubricant with a fixed H-B number and power-law index.

3. Pressure is high at the centre of the bearing and decreases along the radial direction.

4. The pressure is found to increase when the amplitude of the sinusoidal fluid film increases, but only up to an optimum point of time, beyond which the pressure is found to decrease with the increase in amplitude before it changes its course with respect to time.

5. The load capacity of the bearing increases with the increase of time up to an optimum point beyond which it starts decreasing gradually before it changes its nature.

6. The effects of non-Newtonian characteristics on the load capacity is more significant when the amplitude of the sinusoidal motion is very high.

\section{ACKNOWLEDGEMENT}

The authors would like to thank the management of SSN College of Engineering for providing necessary facilities to carry out this work.

\section{REFERENCES}

Chan, T.W and D.G. Baird (2002). "An evaluation of a squeeze flow rheometer for the rheological characterization of a filled polymer with a yield stress", Rheol. Acta, Vol. 41, 245-256.

Elsharkawy, A., S.Z. Kassab and M.M. Nassar (1996). "Lubrication analysis of externally pressurized circular porous bearing", Applied Mathematical Modelling, Vol. 20, 870-876.

Hashimoto, H. and S. Wada (1986). "The effects of fluid inertia forces in squeeze film bearings lubricated with pseudo-plastic fluids", Bulletin of JSME, Vol. 29, 1913-1918.
Jaw-Ren, L. (1999). "Static and dynamic characteristics of externally pressurized circular step thrust bearings lubricated with couple stress fluids", Tribology International, Vol. 32, 207-216.

Jayakaran Amalraj, I., S. Narasimman and A. Kandasamy (2010). "Rheodynamic lubrication of an externally pressurized thrust bearing using Bingham fluid with sinusoidal injection", Proceedings of $4^{\text {th }}$ International Conference on Fluid Mechanics and Fluid Power, IIT Madras, India.

Kandasamy, A. and K.P. Vishwanath (2007). "Rheodynamic lubrication of a squeeze film bearing with inertia effects under sinusoidal squeeze motion using Bingham fluids" Computational and Applied Mathematics, Vol. 26, 381-396.

Kandasamy, A., S. Narasimman and I. Jayakaran Amalraj (2006). "Rheodynamic lubrication of an externally pressurized thrust bearing with Herschel-Bulkkley fluids", Proceedings of the International conference on Iindustrial Tribology, IISc, Bangalore, India.

Kandasamy, A., S. Narasimman and I. Jayakaran Amalraj (2006). "Rheodynamic lubrication of squeeze film bearing with Herschel-Bulkley fluids", Computational Fluid Dynamics Journal, Vol. 14, 436-440.

Meeten G.H. (2005). Flow of soft solids squeezed between planar and spherical surface", Rheol. Acta, Vol. 44, 563-572.

Roy, J.S., S. Padhy and L.K. Bhopa (1993). "Inertia effects in externally pressurized thrust bearing with converging and diverging film using viscoelastic lubricant”, Acta Mechanica, Vol. 96, 1-12.

Sherwood, J.D and D. Durban (1998). "Squeeze-flow of a Herschel-Bulkley fluid", Journal of NonNewtonian Fluid Mechanics, Vol. 77, 115-121.

Usha, R. and P. Vimala (2002). "Curved squeeze film with inertial effects-energy integral approach", Fluid Dynamics Research, Vol. 30, 139- 153.

Whorlow, R.W. (1980). Rheological Techniques, Wiley, New York.

Zhang, J., K.E. Khayat and A.P. Noronha (2005). "Three dimensional lubrication flow of a HerschelBulkley fluid", International Journal of Numerical methods, Vol. 50, 511-530. 